\title{
On the Localisation of Anthocyanin in the Spring Leaves of Some Trees and Shrubs in the Temperate Regions of Japan.
}

by

Tsutsumi Ichimura.

(Professor of Botany in Fourth Higher School, Kanazawa, Eapan.)

The reddening of young leaves just developing in the spring time is so remarkable in the vicinity of Kanazawa that everyman never fails to observe it. Microscopical examinations of the tissues containing red pigment (anthocyanin) evince that they hold the pigment in solution in their cell-sap, which, of course, occupies the vacuole of the cells. My observations on the histological distribution of such periodic anthocyanin have been made on the trees and shrubs in that locality, which turn red most conspicuously.

The localisation of the pigment in the leaves is as follows, the number of genera being 44 in all :-

\begin{tabular}{|c|c|c|c|c|c|c|c|c|}
\hline Names of Plants. & 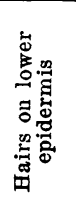 & 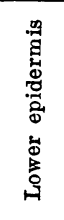 & 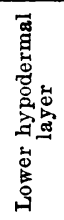 & 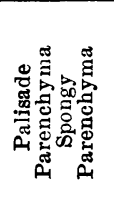 & 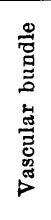 & 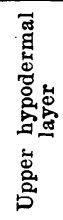 & 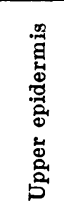 & 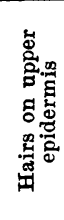 \\
\hline 1. Juglans ( $\bar{O}$ gurumi $)$ & 0 & & + & & & + & & 0 \\
\hline -Juglandaceae. & & & & & & & & \\
\hline 2. Populus (nigra) & & & $(+)$ & & & + & & \\
\hline Salix (Akame-yanagi & & & + & + & & + & & \\
\hline Okawa-yanagi. & & & + & + & & & & \\
\hline Neko-yanagi. & 0 & & + & + & & & & 0 \\
\hline Yamaneko-yanagi). & 0 & & + & + & & + & & 0 \\
\hline -Salicaceae. & & $(+)$ & & & & & & \\
\hline 3. Alnus (Hannoki, & $(+)$ & $(+)$ & & $+(+)$ & & & $(+)$ & 0 \\
\hline $\begin{array}{l}\text { Yama-hannoki). } \\
\text {-Betulaceae }\end{array}$ & 0 & & & $+t_{2}$ & & + & $(+)$ & 0 \\
\hline
\end{tabular}


4. Quercus (Aragashi,

Quercus (Aragash
Akagashi,
Shirakashi,
Nara,
Mizu-nar
Kunugi).
-Fagaceae
Zelkowa (Keyaki)
Celtis-(Enoki)
-Ulmaceae

6. Illicium (Shikimi)

-Magnoliaceae

7. Nandina (Nanten)

- Berberidaceae

8. Cinnamomum

(Kusunoki,

Yabu-nikkei).

Machilus (Tabunoki).

Lindera (Tendai-

-Lauraceae

9. Rosa (Kōshunbara, No-ibara).

Rubus (Momiji-ichigo)

Pirus (Nashi,

Miyama-nanakamado)

Photinia (Kaname-

mochi)

Prunus (Ume, Sakura,

Anzu,

Sumomo).

Cydonia (Boke)

- Rosaceae

10. Wistaria (Fuji)

-Leguminosae

11. Zanthoxylon

(Karasu-zanshō)

-Rutaceae

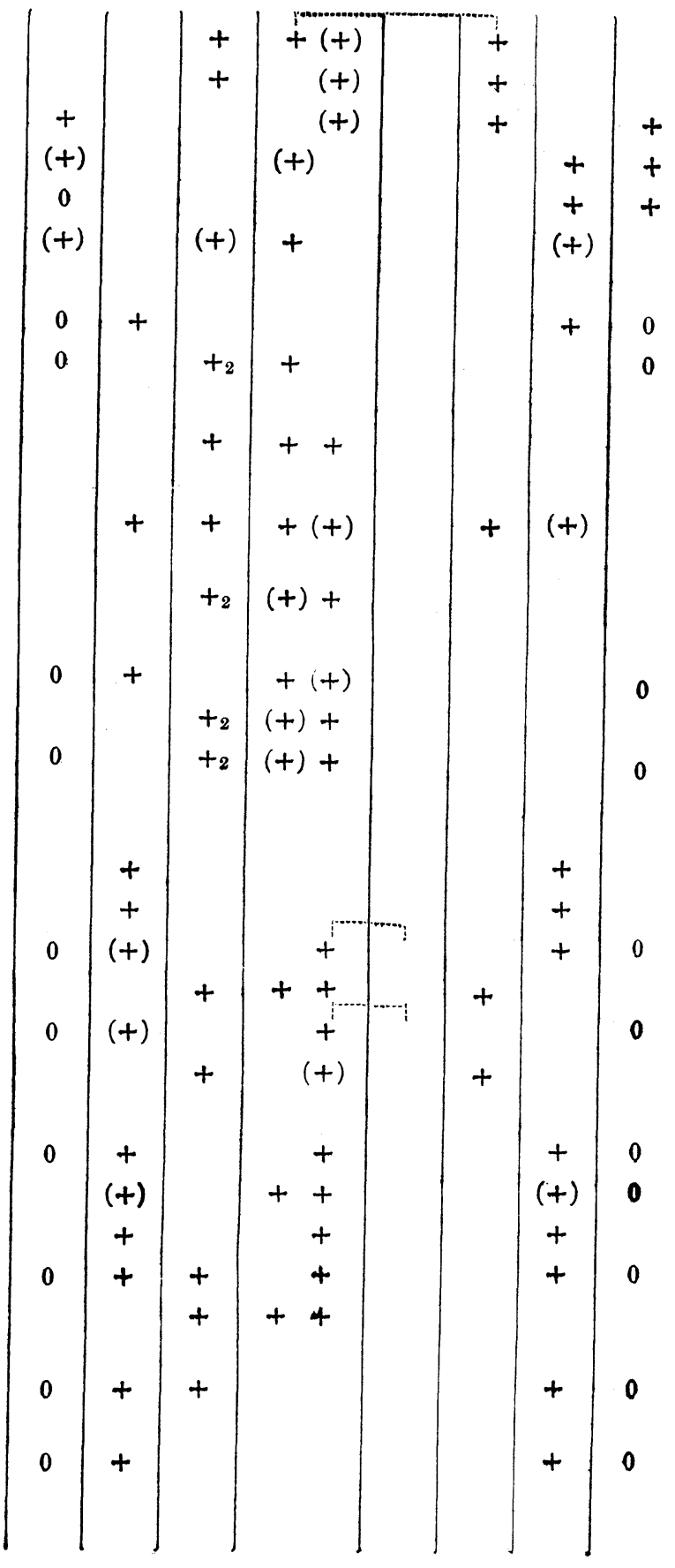


12. Ailanthus (Shinju) -Simarubaceae

13. Cedrela (Chanchin) -Meliaceae

14. Mallotus (Akamegashiwa)

-Euphorbiaceae

15. Khus (Rä-noki, Fußhi-noki, Yamaurushi) -Anacardiaceae

16. Ilex (Mochinoki, A otago) -Aquifoliaceae

17. Euonymus (Masaki) -Celastraceae

18. Acer (Nomura, Tamuke, Tō-kaede) -Aceraceae

19. Sterculia (Aogiri) -Stercaliacea

20. Thea (Tsubaki, Sazankwa) Eurya (Sakaki, Hisakaki) Taonabo (Mokkoku) -Theaceae

21. Stachyurus (Kifuji) Stachyuraceso

22. Lagerstroemia (Saru-suberi) -Lythraceae

23. Punica (Zakuro) -Punioaceae

24. Cornus (Mizuki, Yama-bōōhi)

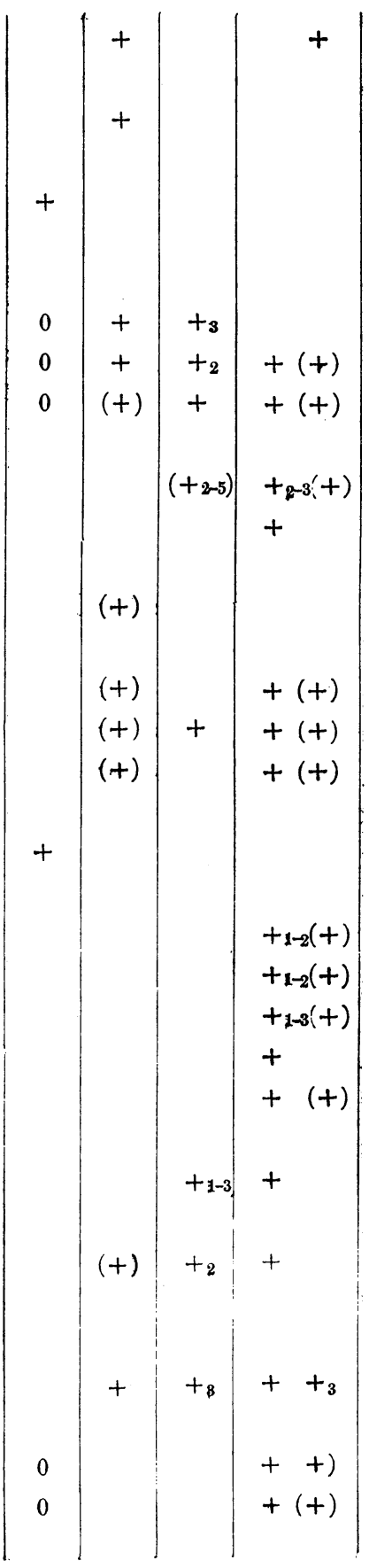

\begin{tabular}{l|c|c}
+ & $(+)$ \\
+ & $(+)$ \\
& & \\
& & \\
& $(+)$ & \\
$(+)$ & $(+)$
\end{tabular}

$+$

(4)

$(+)$ -Cornecere 
25. Leucothoe (Hanahirinoki)

Vaccinum (Natsuhaze)

Tripetaleia (Hotsu-

-Ericaceae

26. Diospyros (Kaki)

-Ebenaceae

27. Symplocos (Sawa-

28. Styrax (Egonoki)

-Styracaceae

29. Osmanthus (Hiiragi, Mokusei)

-Oleaceae

30. Clerodendron(Kusagi) - Verbenaceae

31. Viburnum

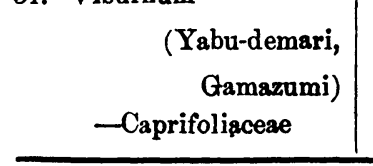

Total

Confined.

(30)

(34)

$+$

$+(+)$
$+(+)$

$1+10$

0

\section{(1)}

$0 \quad(+)+$

$+($

$+(+)$

$(+)(+)$

sujii)

$(+)$

$(+) \quad 0$

$0 \quad(+)$

$+(t)$

0

0

$++(+)$

$+\stackrel{(+)(+)}{+}$

$+$

(0

$\begin{array}{lll}2 & 7\end{array}$

+ Anthocyanin well develops.

(t) Soon it disappears.

0 Hairs present, but no anthocyanin.

I see that anthocyanin is, in a majority of 69 species, localised in the palisade (hypodermal layers when young) and spongy parenchyma in the spring leaves and that it is, in a few of them, confined to epidermis (Rosa, Zanthoxylon, Cedrela, Euonymus etc.) or to hairs (Mallotus, Sterculia). So the distribution of transitory anthocyanin in the young leaves is to be classified as :-

$$
\begin{array}{cccc}
\text { Hairs } & \text { Epidermis } & \text { Mesophyll } & \text { Epidermis and Mesophyll } \\
3 \% & 10 \% & 47 \% & 39 \%
\end{array}
$$

It is also noticeable that the lower epidermis and the lower hypodermal layer are richer in the pigment than the upper ones in the young leaves. 